\title{
What is the relationship between groin pain in athletes and femoroacetabular impingement?
}

\author{
M Ferguson, ${ }^{1} \mathrm{~J}$ Patricios ${ }^{2,3,4}$ \\ ${ }^{1}$ The Centre for Sports Medicine and Orthopaedics, Johannesburg, South Africa; \\ ${ }^{2}$ Morningside Sports Medicine, Johannesburg, South Africa; \\ ${ }^{3}$ Section of Sports Medicine, Faculty of Health Sciences, University of Pretoria, Pretoria, South Africa; \\ ${ }^{4}$ Department of Emergency Medicine, Faculty of Health Sciences, University of the Witwatersrand, \\ Johannesburg, South Africa \\ Correspondence to Dr Jon Patricios, PO Box 1267, Parklands 2121, South Africa; jpat@mweb.co.za
}

Groin symptoms in athletes have been likened to the Bermuda Triangle as sports physicians, orthopaedists, surgeons and therapists alike are easily sucked into a sea of confusing suppositions and assump-tions. ${ }^{1}$ Possibly the greatest advance in the understanding and appreciation of 'sports hernia' has been the recent acknowledgment by a multidisciplinary panel of experts that there is in fact no hernia associated with the condition and they have coined the term 'inguinal disruption'. Unfortunately, although the authors describe a constellation of symptoms, the label again suggests a localised pathology rather than a syndrome

\section{A BROADER FOCUS REQUIRED}

As much as we concentrate our collective efforts on repairing a defect in an anatomical area or treating what we consider to be the primary area of injury, perhaps we should explore and investigate the concept that symptoms are a result of a structural failure of tissue to compensate for abnormal loads created by an imbalance of forces across the groin area. Single or multiple areas of groin injury may represent a failure of the same functional unit. The proposal of a 'pubic joint', distinct from the symphysis pubis, which involves numerous anatomical structures and their resultant forces at different contact sites can explain the multiple pathologies that exist in this condition ${ }^{3}$ This refers to the musculoskeleton of the pelvis outside of the hip joint arranged symmetrically around and including the symphysis.

This would include all the previously described entities of external oblique tears, posterior wall deficiencies, conjoined tears, traction neuropathy, adductor tendinopathy, osteitis pubis, rectus ethesopathy, etc. Having all these different terms explains why at present there are no generally accepted diagnostic criteria for the different pathologies causing groin pain.

Recent dissection and radiographic publications regarding the anatomy of the area have confirmed the complex association of these structures to the symptoms athletes typically experience when sprinting, kicking and pivoting ${ }^{34}$ 


\section{ARTHROSCOPIC ADVANCES}

Over the past two decades the increasing use of hip arthroscopy in orthopaedics has identified femoroacetabular impingement (FAI) as a major cause of hip pain, reduced range of movement and decreased performance. As described by Cakic, ${ }^{5} \mathrm{FAl}$ is a hip condition caused by abnormal contact between the femur and the acetabulum due to subtle developmental disorders of the hip. Recurrent impingement of the intervening tissue can lead to tears of the acetabular labrum and chondral surface.

\section{AND RADIOLOGICAL REFINEMENT}

Clinical tests of impingement are described but the diagnosis is usually based on at least eight different radiological signs. These include an $\alpha$ angle greater than $55^{\circ}$ as indicative of cam lesion and the pincer lesion being identified by (among other radio-logical features) the presence of a 'crossover sign' on an appropriately taken anteroposterior pelvic X-ray. These represent morphological changes that have been found to have a prevalence of $14 \%$ in asymptomatic volunteers, $72 \%$ in symptomatic and asymptomatic elite soccer players and as high as $94 \%$ in athletes with adductor-related groin pain. ${ }^{6}$ The possibility of a relationship between FAI and groin pain cannot be ignored; however, clear criteria for the diagnosis of FAI remain controversial and no gold standard exists at present. ${ }^{7}$

\section{BIOMECHANICAL HYPOTHESES}

Some cadaveric biomechanical studies have attempted to explain this relationship by simulating a decreased head-neck offset that produced increased motion at the symphysis pubis, proposing that this may be a contributing factor to the development of inguinal disruption. However, significant contact has to occur between the offset and the acetabular rim to cause this increased symphyseal movement. This is likely to lead to a very high incidence of labral lesions in the subset of athletes with FAl and inguinal disruption, which is not evident clinically.

Kennedy ${ }^{8}$ studied the altered hip and pelvic biomechanics associated with FAI with walking, and concluded that the decreased abduction and increased frontal pelvic movement is unlikely to be due to the contact between the head-neck junction but rather a gait adaptation representing a different motion strategy, characterised by differences in the recruitment of hip joint muscles. On this basis, as a compensatory pattern, an overload or imbalance involving the musculature of the anterior and medial compartment of the pelvis could cause tissue failure of the type often described in inguinal disruption. ${ }^{9}$

Conversely the pathomechanics of the relationship between FAI and inguinal disruption could be explained as arising from the latter condition in the presence of an underlying asymptomatic FAl. Owing to the nature of movements carried out by these athletes, tremendous torque forces occur across the pubic joint. Common findings are a facilitated quadratus lumborum and overactive adductors which can in turn lead to inhibition, weakness or tightness of the psoas and ultimately delayed activation of the transversus abdominus and pelvic floor. The consequence hereof may be anterior overload with failure of the rectus abdominus and external oblique with a resultant net anterior pelvic tilt. This overcoverage of the pelvis due to the anterior pelvic tilt in a susceptible 
athlete who has a reduced acetabular distance could cause symptoms of FAI and ultimately labral pathology.

\section{ACKNOWLEDGING A MECHANICAL ASSOCIATION}

Acknowledgement of the causative affect of altered mechanics around the pubic joint could explain the reported reduction in the incidence of inguinal disruption due to preventative programmes and the success of conservative treatment with fewer athletes requiring surgery. ${ }^{3}$

Unfortunately, due to the coexistence of FAl and inguinal disruption in a certain subset of athletes, the treating physician is faced with the dilemma of how to differentiate the signs of each condition. Recent retrospective outcome studies of surgery for these athletes have recognised poor results after surgery for FAl or inguinal disruption alone when compared with those patients in whom surgery was per-formed on the FAI and inguinal area simultaneously. ${ }^{10}$

This again highlights the need for exploring associations between FAl and inguinal disruption as well as well designed prospective outcome studies that would allow us as physicians to identify the best individual treatment options for both conditions. Probing common risk factors of inguinal disruption and FAl rather than focusing on isolated local injury may well be the answer to demystifying the clinician's equivalent of the treacherous Bermuda triangle.

Contributors MF researched and drafted the original paper. JP reviewed, expanded and reformatted the article.

\section{Competing interests None.}

Provenance and peer review Not commissioned; internally peer reviewed.

\section{REFERENCES}

${ }^{1}$ Bizzini M. The groin area: the Bermuda triangle of sports medicine? Br J Sports Med 2011;45:1.

${ }^{2}$ Sheen AJ, Stephenson BM, Lloyd DM, et al. Treatment of the sportsman's groin': British Hernia Society's 2014 position statement based on the Manchester Consensus Conference. Br J Sports Med 2014;48:1079-87.

${ }^{3}$ Meyers WC, McKechnie A, Philippon MJ, et al. Experience with 'Sports Hernia' spanning two decades. Ann Surg 2008;248:656-65.

${ }^{4}$ Omar IM, Zoga AC, Kavanagh EC, et al. Athletic pubalgia and 'sports hernia': optimal MR imaging technique and findings. Radiographics 2008;28:1415-38.

${ }^{5}$ Cakic J, Patricios J. Femoroacetabular impingement: prevention or intervention? The sports physician's quandary. Br J Sports Med 2014;48:1073-74.

${ }^{6}$ Weir A, de Vos RJ, Moen M, et al. Prevalence of radiological signs of femoroacetabular impingement in patients presenting with long-standing adductor-related groin pain. Br J Sports Med 2010;45:6-9. 
${ }^{7}$ Standaert CJ, Manner PA, Herring SA. Expert opinion and controversies in musculoskeletal and sports medicine: femoroacetabular impingement. Arch Phys Med Rehabil 2008;89:890-3.

${ }^{8}$ Kennedy MJ, Lamontagne M, Beaulé PE. Femoroacetabular impingement alters hip and pelvic biomechanics during gait. Gait Posture 2009;30:41-4.

${ }^{9}$ Bedi A, Dolan M, Leunig M, et al. Static and dynamic mechanical causes of hip pain. Arthroscopy 2011;27:235-51.

${ }^{10}$ Larson CM, Pierce BR, Giveans MR. Treatment of athletes with symptomatic intra-articular hip pathology and athletic pubalgia/sports hernia: a case series. Arthroscopy 2011;27:768-75. 